\title{
Star formation in the Galactic bulge: the role of collisions
}

\author{
Mark Cropper and Dave Vande Putte \\ Mullard Space Science Laboratory, University College London, Holmbury St Mary, Dorking, \\ Surrey RH5 6NT, UK \\ email: msc@mssl.ucl.ac.uk
}

\begin{abstract}
Prompted by the possibility that we have observed star formation triggered by globular cluster and dwarf spheroidal transits through galactic disks, we have examined kinematic evidence as to whether the superstar clusters in the Galactic bulge could have been formed from such transits. From their trajectores, we cannot exclude such a possibility. We note also that the high frequency of these transits may have generated rapid star formation at early times, and that remnant cluster cores may themselves nucleate further star formation.
\end{abstract}

Keywords. galaxies: evolution, galaxies: star clusters, Galaxy: bulge, stars: formation.

\section{Globular Cluster Transits through the Bulge}

It is possible that star formation has, in some particular instances been induced by collisions between existing star clusters and gas rich material in the disk or bulges of galaxies. Examples include the star formation complex NGC346 in the SMC which is associated with an older globular cluster BS90 (Nota et al. 2006) and the burst of star formation just outside the disk of NGC4559 which may have been induced by the passage of a dwarf spheroidal galaxy (Soria et al. 2004).

There are $>155$ Globular Clusters (GCs) in the Galaxy recorded in the most recent (2003) catalog of Harris (1996). For a subset of 48, there are proper motions and radial velocities (Allen, Moreno \& Pichardo 2006) which allow orbits to be calculated.

We have calculated the orbits for 4 potentials. Given the starting positions and velocities from above, our code integrates the equations of motion numerically in cylindrical coordinates. The code provides the time and position of Galactic plane transits for each of these GCs. These calculations indicate that a significant fraction of GCs have transited within $4 \mathrm{kpc}$ of the Galactic centre $>400$ times. The total number of transits for these 48 GCs is $\sim 9400$, and scaling to the full Harris (2003) sample, this increases to $\sim 30000$, or $\sim 0.5 \mathrm{Myr} /$ transit on average for the surviving system of GCs.

We have now examined the effect on stars within the Galactic bulge resulting from a GC transit. The GC is modelled using a Plummer potential, orbiting on its trajectory within the Galactic potential. We identify the stars that will encounter the GC, with a range of impact parameters. We then propagate the trajectories of these stars in the time-varying Galaxy+GC potential and observe their perturbed motions. These are shown in Figure 1 (left). Figure 1 (right) indicates that there is a substantial short-term concentration (focussing) of the stars. It is this focussing which may induce star formation should it take place in a location where there is sufficient gas for dissipative processes to take over.

\section{Effects on the Bulge: Super Star Clusters}

Given the above focussing, we conjecture that particular forms of star formation, such as that for super star clusters, may be induced by GC transits. We have therefore examined whether the super star clusters in the bulge, the Arches cluster and the Quintuplet may have been formed by a GC transit. 

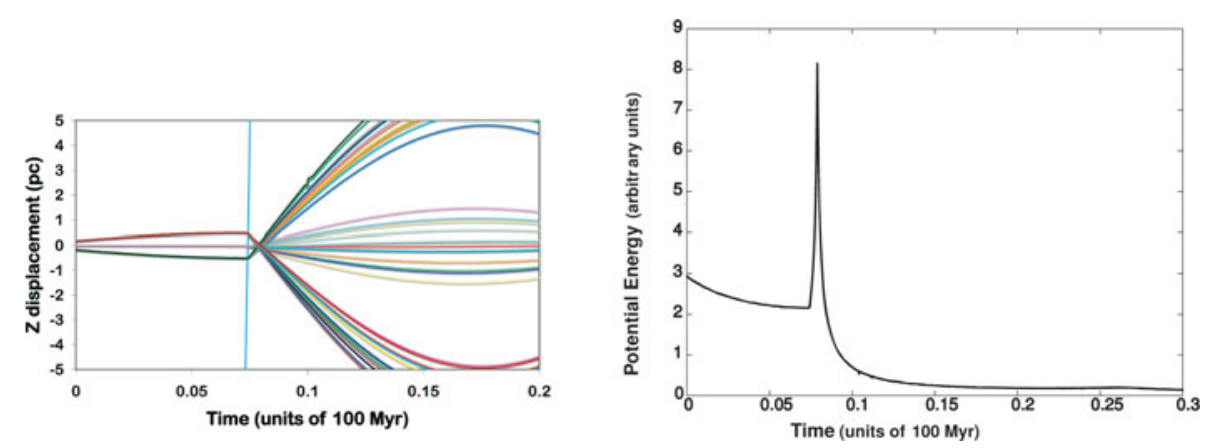

Figure 1. Left: the perturbed $z$ displacements of stars in a cube with dimensions 1 pc interacting with a GC of mass $5 \times 10^{5} \mathrm{M}_{\odot}$ and core radius $0.5 \mathrm{pc}$ (blue quasi-vertical line). Right: the gravitational focussing caused by the interaction (inverse of mean separation).

We have examined the motions of the 48 clusters in the Allen et al. (2006) list, using a Monte-Carlo technique to explore the trajectory space permitted by the observational errors (positions and motions) and by the different Galactic potentials. Current observational uncertainties provide only a weak test, such that a significant number of the GCs could have passed in the vicinity of these clusters if the star formation time is $<30 \mathrm{Myr}$ (the duration suggested by Wallin, Higdon \& Stavely-Smith 1996).

We then considered a more general case for all of the GCs in the Harris (2003) sample. Without access to the velocities for those outside of the Allen et al. (2006) sample, we can nevertheless place limits by considering simply the escape velocity of the Galaxy potential, and consider whether it would be possible for the GCs to have reached the location of the super star clusters from their current position given the total time tt above. For this simple test, only 15 GCs of the total 155 can be eliminated as possible candidates for inducing star formation in Arches.

\section{Bulge Formation and Induced Star Formation at Early Epochs}

GC transits through the bulge will contribute to some level of heating of the bulge. The effects on the GCs themselves have been considered by several authors, such as Aguilar et al. (1988), who concluded that the GC system has been significantly diminished through bulge shocking and dynamical friction and that these dissociated GCs have contributed some (relatively small) fraction of the spheroidal component. At early times the rate of transits will have been much higher and transits of GCs and Dwarf Spheroidals could have stimulated extremely widespread and coordinated bursts of star formation, driving gas from the bulge and quenching further star formation. Moreover, the cores of disrupted clusters may still retain their existance, even though they may be composed mainly of stellar remnants, These may themselves nucleate star formation through their gravitational perturbative effects as they pass through regions where conditions for star formation are suitable.

\section{References}

Aguilar, L., Hut, P., \& Ostriker, J. P., 1988, ApJ, 335, 218

Allen, C., Moreno, M., \& Pichardo, B., 2006, ApJ, 652, 1150

Harris, W. E., 1996, $A J, 112,487$ (see www.physics.mcmaster.ca/Globular.html for 2003 version)

Nota, A., et al. 2006, ApJ, 640, L29

Soria, R., Cropper, M., Pakull, M., Mushotzky, R., \& Wu, K., 2005, MNRAS, 356, 12

Wallin, J. F., Higdon, J. L., \& Stavely-Smith, L., 1996, ApJ, 459, 555 